\title{
The accuracy of noninvasive total hemoglobin measurement in critically ill patients
}

R. Oishi, M.D., S. Obara, M.D., M. Hasegawa, M.D., T. Hakozaki, M.D., Y. Iseki, M.D., M. Murakawa, M.D. Fukushima Medical University, Fukushima, Japan.

\section{Background and Goal of Study}

In critically ill patients, monitoring total hemoglobin concentrations $(\mathrm{tHb})$ is essential. The Pronto Pulse CO-Oximeter (Masimo Corporation, Irvine, $\mathrm{CA}$ ) monitors $\mathrm{tHb}$ noninvasively $(\mathrm{SpHb})$. Although good correlations between the $\mathrm{SpHb}$ and invasively measured $\mathrm{tHb}$ are reported in healthy patients, the relationship between the two indices in critically ill patients is not well investigated.

The aim of this study was to evaluate the accuracy of $\mathrm{SpHb}$ measurements in critically ill patients.

\section{Materials and Methods}

We enrolled patients admitted to the ICU of Fukushima Medical University Hospital, from August 2016 to February 2017. The study was approved by the ethical committee at Fukushima Medical University, and all patients or their family provided written informed consent.

Blood was collected during routine blood examinations and analyzed using a cellular analysis system (UniCel DxH 800; Beckman Coulter, Brea, CA). Within 30 minutes of the invasive blood sampling, $\mathrm{SpHb}$ values were recorded for a comparative study.

The data were analyzed using Spearman rank-order correlation and Bland-Altman analysis. A $p$ value of $\leq 0.05$ was considered significant.

\section{Results and Discussion}

Of 120 measurements from 34 patients, 88 measurements were successfully obtained. Table 1 shows the characteristics of enrolled patients.

Figure 1 shows the correlation between $\mathrm{tHb}$ and $\mathrm{SpHb}$. The $\mathrm{R}$ value was $0.68(\mathrm{P}<0.001)$.

To assess the agreement between $\mathrm{SpHb}$ to $\mathrm{tHb}$, Bland-Altman plot was applied(figure 2). The bias was $1.43 \mathrm{~g} / \mathrm{dL}$ (95\% confidence interval, $1.17-1.68 \mathrm{~g} / \mathrm{dL}$ ), and $95 \%$ limits of agreement (LOA) was $-1.39-4.24 \mathrm{~g} / \mathrm{dL}$.

In our study, the accuracy of $\mathrm{SpHb}$ measurement was acceptable and the LOA was almost comparable to that of other recent studies ${ }^{1}$ $(-2.90-3.26 \mathrm{~g} / \mathrm{dL})$. Several studies have demonstrated that the accuracy of $\mathrm{SpHb}$ measurements is significantly reduced in the presence of vasoconstriction, which is observed in critically ill patients. This probably explains the relatively wide LOA observed in the present study.

Nevertheless, SpHb levels could not be measured in $20 \%$ (22) of the cases. Phillips et al. ${ }^{2}$ have reported that hypoxia and hypothermia were predictors of failure of the device. Patients in the ICU tend to experience such conditions; therefore, we should be careful with clinical decisions based on this technology.

\section{Conclusion}

The accuracy of $\mathrm{SpHb}$ measurement was acceptable, but clinicians should carefully consider the wide LOA and the probability of capturing failure in critically ill patients before making clinical decisions based on this measurement.

Table 1. Characteristics of Enrolled Patients

\begin{tabular}{cc}
\hline Variable & Mean \pm SD [range] \\
\hline Age (years) & $67.2 \pm 10.1[42-85]$ \\
Male gender (\%) & 44.1 \\
Weight $(\mathrm{kg})$ & $54.3 \pm 14.4[35-96]$ \\
Height $(\mathrm{cm})$ & $156.2 \pm 11.3[132-176.6]$ \\
BMI $\left(\mathrm{kg} / \mathrm{m}^{2}\right)$ & $22.1 \pm 4.5[14.4-35.7]$ \\
\hline Reason for ICU admission & $\mathrm{n}(\%)$ \\
\hline Cardiovascular surgery & $21(61.8)$ \\
Major open surgery & $6(17.6)$ \\
Pneumonectomy & $2(5.9)$ \\
Pneumonia & $2(5.9)$ \\
Others & $3(8.8)$ \\
\hline
\end{tabular}
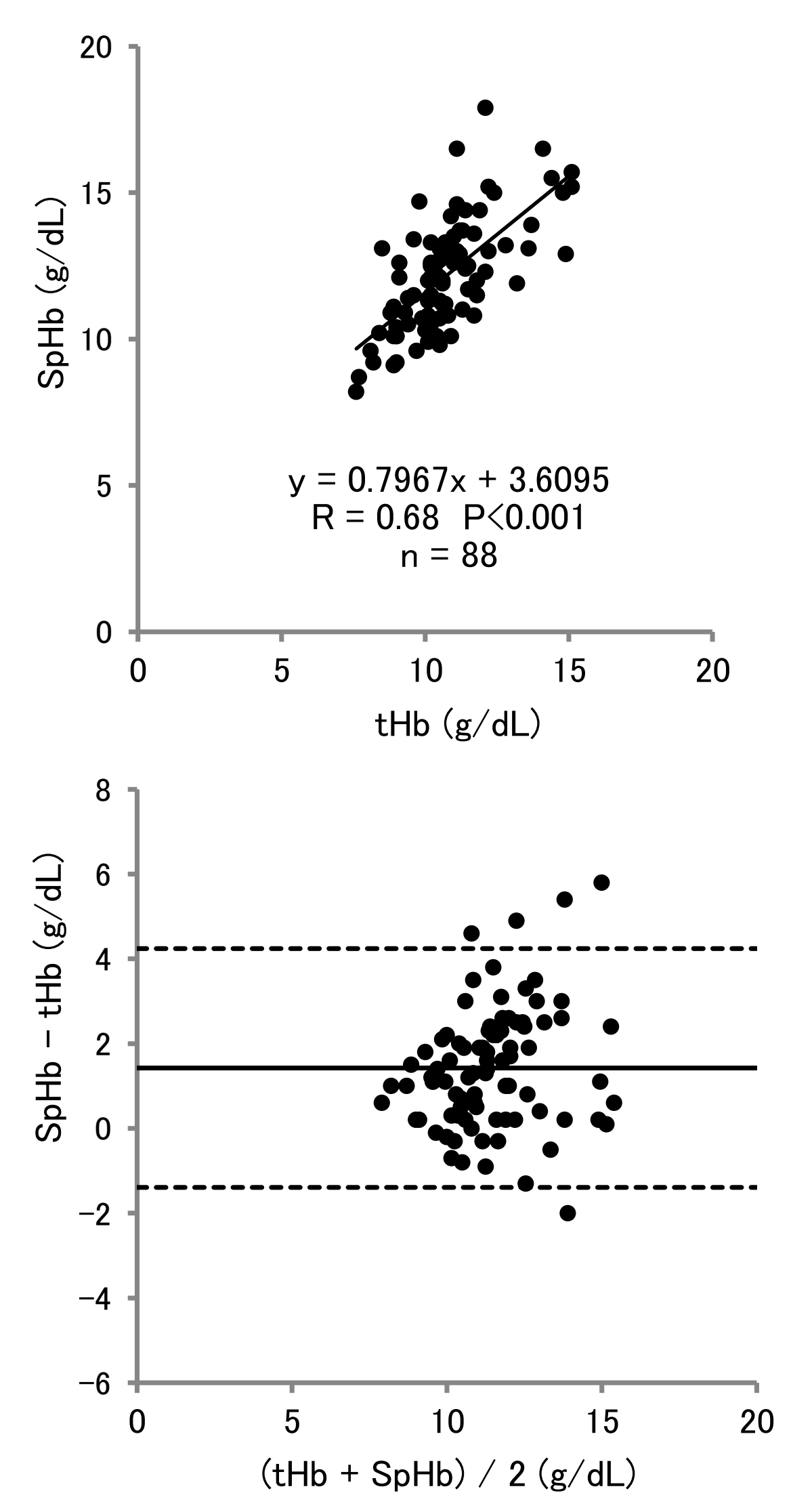

Figure 1. The Relationship Between $\mathrm{THb}$ and $\mathrm{SpHb}$

Observed noninvasive and continuous hemoglobin monitoring $(\mathrm{SpHb})$ measurements plotted against laboratory $\mathrm{CO}-\mathrm{Oximeter} \mathrm{Hb}$ $(\mathrm{tHb})$ values.

Figure 2. Bland-Altman Plot for Overall Data

Relationship between the observed differences of hemoglobin measured by laboratory $\mathrm{CO}-\mathrm{Oximeter}(\mathrm{tHb})$ and noninvasive and continuous hemoglobin monitoring $(\mathrm{SpHb})$ and the mean of the 2 measurements.

\section{References}

1. Anaesth Intensive Care. 2015; 341-350

2. J Surg Res. 2015; 257-62 